Recepción: 15 / 04 / 2017

Arquitectura

Aceptación: 01 / 05 / 2017

Artículo Científico

Publicación: 15 / 05 / 2017

\title{
Utilización del chip de plástico como material alternativo en la construcción de viviendas en Machala
}

Use of the plastic chip as an alternative material in the construction of houses in Machala

Usando o chip de plástico como um material alternativo na construção de habitação em Machala 


\section{Resumen}

La contaminación resultante de los desechos plásticos dentro de los cuales está el PET, un plástico que tiene un periodo de vida de aproximadamente 500 años, obligó a tomar medidas medioambientales para disminuir la aportación de estos desechos al medioambiente.

En el presente estudio de caso, se utilizó el PET como material alternativo en la construcción de viviendas, ya que se ha estado desarrollando y difundiendo en los últimos años en países como Argentina, Colombia, México, inclusive en nuestro país, que es un nuevo método de crear arquitectura sostenible, disminuyendo la contaminación del ambiente y fomentando a la vez la posibilidad de realizar construcciones con ese tipo de materiales. Para el desarrollo de este trabajo fue necesario en primer lugar la investigación de los sitios de acopio de las botellas plásticas, los equipos necesarios para la trituración de las botellas para obtener el CHIP DE PLASTICO, para luego hacer las mezclas de acuerdo a los diseños de la formula maestra para la obtención del bloque no portante, hueco de 7 y $10 \mathrm{~cm}$. Lo antes descrito es la base para la construcción de viviendas con bloques de PET, reutilizando este desecho plástico al proceso constructivo dentro de la ingeniería civil y disminuyendo el impacto ambiental que genera la construcción tradicional.

Palabras clave: Botellas de plástico; reciclaje; polietileno tereftalato (PET); bloques; medio ambiente; desechos; recicladoras; chamberos; chip de plástico; mezcla; formula maestra; vivienda; arquitectura sostenible. 
Judy M. Flores-Cherrez; Brick L. Reyes-Pincay; Saturnino A. Carabajo-Ayala; María B. Alarcón-Valencia

\begin{abstract}
The pollution from plastic waste within is PET, a plastic that has a lifetime of approximately 500 years, forced to take environmental measures to reduce the contribution of these wastes on the environment.

The present case of study, is focuses for PET use how to alternative in built houses with development in last years in the next countries Argentina, Colombia, México, include in our country is a new method to build stable architecture, avoiding contamination of the environment and promoting both the possibility of constructions with such materials. This work is walking in the first place in a investigation in recycle stores of plastic bottles, equipment necessary for crushing the bottles to get the CHIP PLASTIC, then make the mixtures according to the designs of the master formula for obtaining non-bearing block, side 7 and 10 centimeter. All after wrote is used of base for build houses with a PET blocks, reused this recycle plastic to process inside of Civil Engineer and reducing the environmental impact generated by traditional construction.
\end{abstract}

Key words: Plastic bottles; recycling; polyethylene terephthalate (PET); blocks; environment; waste; recycling; chamberos; plastic chip; mix; master formula; housing; sustainable architecture. 


\section{Resumo}

A poluição proveniente dos resíduos plásticos é o PET, um plástico que tem uma vida útil de cerca de 500 anos, obrigado a tomar medidas ambientais para reduzir a contribuição destes resíduos para o ambiente.

O presente caso de estudo, é focos para uso de PET como alternativa em casas construídas com desenvolvimento nos últimos anos nos próximos países Argentina, Colômbia, México, incluem em nosso país é um novo método para construir uma arquitetura estável, evitando a contaminação do meio ambiente E promovendo tanto a possibilidade de construções com tais materiais. Este trabalho está caminhando em primeiro lugar em uma investigação em lojas de reciclagem de garrafas de plástico, equipamentos necessários para esmagar as garrafas para obter o CHIP PLÁSTICO, em seguida, fazer as misturas de acordo com os desenhos da fórmula mestre para a obtenção de nãorolamento bloco, lado 7 e 10 centímetros. Tudo depois escrito é usado de base para construir casas com blocos de PET, reutilizando este plástico reciclado para processar dentro de Engenheiro Civil e reduzindo o impacto ambiental gerado pela construção tradicional.

Palavras chave: Garrafas plásticas; reciclando; Tereftalato de polietileno (PET); Blocos; meio Ambiente; desperdício; reciclando; Camberos; Plástico chip; misturar; Fórmula-mestre; habitação; Arquitetura sustentável. 
Judy M. Flores-Cherrez; Brick L. Reyes-Pincay; Saturnino A. Carabajo-Ayala; María B. Alarcón-Valencia

\section{Introducción.}

La utilización de los materiales de construcción por la demanda de vivienda en nuestro país se hace en grandes cantidades, por lo que deben provenir de materias primas abundantes y de bajo costo (Parrales, 2016). Por ello, la mayoría de los materiales de construcción se elaboran a partir de materiales de gran disponibilidad como arena, arcilla o piedra, que no son renovables.

Es conveniente que los procesos de manufactura requeridos consuman poca energíay no sean excesivamente elaborados. Los materiales de construcción tienen como característica común el ser duraderos, dependiendo de su uso, además de satisfacer otros requisitos tales como la dureza, la resistencia mecánica, la resistencia al fuego, o la facilidad de limpieza.

Por norma general, ningún material de construcción cumple simultáneamente todas las necesidades requeridas: la disciplina de la construcción es la encargada de combinar los materiales para satisfacer adecuadamente dichas necesidades (Parrales, 2016).

Con objeto de utilizar y combinar adecuadamente los materiales de construcción los proyectistas deben conocer sus propiedades (Parrales, 2016). Los fabricantes deben garantizar unos requisitos mínimos en sus productos, que se detallan en hojas de especificaciones (Parrales, 2016). Entre las distintas propiedades de los materiales que se encuentran según Parrales (2016):

Densidad: Relación entre la masa y el volumen. Higroscopicidad: Capacidad para absorber el agua.

Coeficiente de dilatación: Variación de tamaño en función de la temperatura.

Conductividad térmica: Facilidad con que un material permite el paso del calor. 
Resistencia mecánica: Capacidad de los materiales para soportar esfuerzos. Elasticidad:

Capacidad para recuperar la forma original al desaparecer el esfuerzo.

Plasticidad: Deformación permanente del material ante una carga o esfuerzo. Rigidez: La resistencia de un material a la deformación.

Una de las mejores soluciones planteadas para mermar el impacto ambiental, es el reciclaje, proceso por el cual se recolecta los materiales de uso común y se transforman para el mismo u otro uso, evitando incurrir a usar más materia prima. El reciclado es la mejor forma de evitar la extracción de materias primas (Gaggino, Arguello, \& Berretta, Doc Player).

Las legislaciones de muchos países trabajan en la necesidad de proteger el medio ambiente presionando desde lo económico, condicionando a que "quien contamina, paga", con diversos grados de éxito (Gaggino, Arguello, \& Berretta, Doc Player).

En este trabajo de investigación se aplica la tecnología para la construcción en reciclando residuos plásticos urbanos, con el objetivo ecológico de colaborar en la reducción de la cantidad de los mismos (Gaggino, Arguello, \& Berretta, Doc Player).

En el mundo se hacen esfuerzos por implementar el reciclado específicamente en la fabricación de bloques o ladrillos para viviendas con PET, en Latinoamérica: México, Colombia, Venezuela, Brasil, Argentina, Nicaragua han avanzado en este proceso de reciclado, con avances considerables, no sólo en la investigación sino en la puesta en práctica del uso del PET en la construcción como una alternativa ecológica.

El presente estudio busca elaborar bloques utilizando el chip de plástico como material alternativo para la construcción de viviendas económicas y ecológicas en la ciudad de Machala. 
Judy M. Flores-Cherrez; Brick L. Reyes-Pincay; Saturnino A. Carabajo-Ayala; María B. Alarcón-Valencia

\section{Materiales y métodos.}

El presente trabajo está enmarcado dentro de un proceso metodológico como herramienta para el desarrollo de las posibles estrategias de solución a la problemática ambiental con alternativas para la utilización de las botellas de plástico, convertidas en el CHIP DE PLASTICO, para elaborar elementos arquitectónicos como bloques para paredes (Zavala, 2015). Dichas herramientas surgen de un proceso de etapas lógicas de investigación el cual se define para tener una comprensión y análisis profundo del problema y así, que éste permita dar una respuesta apropiada; dicha estrategia se plantea de la siguiente manera (Zavala, 2015).

\section{Metodología usada}

Las bases metodologías usadas para este Estudios de caso fueron:

\section{Diseño experimental}

\section{Tipo de Investigación:}

Se utilizó el método descriptivo, en primer momento decidí elegir el tema a estudiar, procediendo de limitarlo, descartamos información escrita y oral acerca de alternativas para la construcción con base en material reciclable, y para esto fue necesaria la recopilación de datos.

Inicié haciendo uso de la investigación documental, entendiéndose a esta como búsqueda de información de documentos, revistas, artículos de divulgación, y libros que den cuenta del tema investigado. 
Según Cerda (1998) una de las funciones principales de la investigación descriptiva es la capacidad para seleccionar las características fundamentales del objeto de estudio y su descripción detallada de las partes, categorías o clases de objeto.

Es experimental porque se obtiene a través de los diseños y ensayos el comportamiento del PET como materia prima para bloques de mampostería. Ya que el investigador actúa directamente sobre el objeto de estudio (Cerda: Investigación experimental), teniendo que experimentar para probar su hipótesis.

\section{Diseño de la investigación}

Consta de investigación documental, de campo y experimental

Investigación Documental: Recopilación de la información contenida en libros, revistas, artículos académicos.

Investigación de Campo: Seguimiento al proceso de reciclado del plástico con visitas al sitio donde se recepta (compra) los desechos plásticos para enviarlos al reciclado.

Investigación experimental: Elaboración de bloques a partir de diseños usando el Chip de plástico como agregado. 
Judy M. Flores-Cherrez; Brick L. Reyes-Pincay; Saturnino A. Carabajo-Ayala; María B. Alarcón-Valencia

\section{CUADRO DE CATEGORÍAS, DIMENSIONES, INSTRUMENTOS Y UNIDADES DE ANÁLISIS (CDIU).}

\begin{tabular}{llll}
\hline CATEGORIAS & DIMENSIONES & INSTRUMENTOS & UNIDADES \\
& & & DE \\
& & & ANÁLISIS \\
\hline SOCIALES & Demanda de vivienda & INEC & Machala \\
ECONOMICAS & Viviendas de bajo costo & PRESUPUESTO & Bloque \\
AMBIENTALES & Material no contaminante & RECICLADORAS & Botellas \\
& alternativo para la & & \\
& construcción. & &
\end{tabular}

Cuadro $N^{\circ}$ 1.- Cuadro de Categorías, dimensiones, instrumentos y unidades de análisis (CDIU)

\section{DESCRIPCIÓN DE LAS UNIDADES DE ANÁLISIS}

\section{Categorías}

Las categorías se extrajeron del árbol del problema donde se enumeran las causas del problema:

\section{Sociales}

En el último censo poblacional del 2010 INEC se registra una población de 14'306.876 habitantes para nuestro país Ecuador con una tasa de acumulación en $1.52 \%$ al año.

De las estadísticas obtenidas del censo 2010 INEC, se obtiene que existe un déficit de parte de la población de un $34.20 \%$ de viviendas, lo que significa que la cantidad de vivienda aún no está satisfecha. 
Tenencia de vivienda 2010 Hogares

$\%$

$\begin{array}{llc}\text { Propia y totalmente pagada } & 1.786,005 & 46,9 \% \\ \text { Arrendada } & 816,664 & 21,4 \% \\ \text { Prestada o cedida (no pagada) } & 489,213 & 12.80 \% \\ \text { Propia (regalada, donada, heredada } & 402,891 & 10,6 \% \\ \text { Propia y la está pagando } & 249,160 & 6.5 \% \\ \text { Por servicios } & 59,145 & 1,6 \% \\ \text { Anticresis } & 7,470 & 0.2 \% \\ \text { Total } & 3.810,548 & 100 \%\end{array}$

\section{Cuadro $N^{\circ}$ 2.- Tenencia de vivienda de los hogares en Ecuador}

Al existir un déficit de vivienda del $34.2 \%$ (entre arrendadas y prestada o cedida), se hace necesario bajar el costo de las viviendas.

La ventaja económica también se obtiene a causa de reintroducir los desechos en el ciclo industrial-comercial; así los materiales reciclados son normalmente más baratos no por su precio en sí, sino porque el constructor debe pagar por deshacerse de él y luego pagar por conseguir algo similar por la obra a realizar

\section{Ambientales}

Disminuir la contaminación ambiental por plástico al transformar y reutilizar dichas botellas de plástico en un material alternativo para la construcción. 
Judy M. Flores-Cherrez; Brick L. Reyes-Pincay; Saturnino A. Carabajo-Ayala; María B. Alarcón-Valencia

\section{Dimensiones}

El dimensionamiento del estudio del caso se circunscribe a aportar con material alternativo para la vivienda a la demanda de vivienda en el Ecuador, los medios o alternativas para la construcción de viviendas accesibles, para lo cual se utilizan materias primas como arena, cemento y aditivos, el costo del bloque obtenido se usa para establecer las conclusiones de la factibilidad del uso del PET como material alternativo para la construcción.

\section{Instrumentos}

Los instrumentos son usados para conseguir la información sobre las variables dimensionadas y que a la vez dan respuestas a las categorías determinada en la matriz CDIU, para el presente caso de estudio por estar enfocado a una alternativa social, económica y ambiental, se utilizan básicamente de tres tipos de instrumentos:

Estadística: Datos de la vivienda en el Ecuador y Machala.

Documental: Obtuve información sobre los usos de esta tecnología en el Mundo y Local.

Diseño y Producción: Se elaboró el diseño de dos tipos de mezcla A y B con la siguiente proporción:

Mezcla A:Pet $0.0075 \%$, Cemento 0.0079\%, Arena 0.0000094\%, Aguas 0.00000044\%

Mezcla B:Pet $0.0091 \%$, Cemento 0.014\%, Arena 0.000011\%, Agua 0.0000076\%. 


\section{Unidades de análisis}

En la matriz obtenida CDIU se detallan las fuentes de obtención de la información requerida, para el dimensionamiento de las variables, obtenidas de artículos en internet, INEC y otros trabajos desarrollados en este campo del reutilizado de botellas plásticas de PET y su aplicación como material alternativo de la construcción.

\section{Gestión de datos}

Los datos iníciales del déficit de vivienda corresponden al último censo poblacional del 2010, como información oficial de la tenencia de vivienda propia en el Ecuador.

De acuerdo a la estadística del INEC, los resultados del último censo de población y vivienda (2010) son 75.473 viviendas en Machala.

Los materiales utilizados: polietileno-Tereftalato, procedente de envases descartables de bebidas; y films de plásticos varios: polietileno, polipropileno biorientado y policloruro de vinilo, procedentes de embalajes de alimentos reciclados. (Zavala, 2015)

Estos residuos se trituran y se incorporan a una mezcla de cemento Portland común, agua y un aditivo químico (Zavala, 2015). Con esta mezcla se fabrican ladrillos, bloques de pared y placas de ladrillos; que se aplican en cerramientos no estructurales de viviendas (Zavala, 2015).

La cuantía de cemento es de 224,5 kg/m3 en el caso del ladrillo (sección bruta); y 103 kg/m3 en el caso del bloque para muro (sección bruta) (Zavala, 2015). El aditivo químico se agrega al agua de mezclado, en un porcentaje del 0,5\% del peso del cemento (Zavala, 2015). Cuando esta mezcla 
Judy M. Flores-Cherrez; Brick L. Reyes-Pincay; Saturnino A. Carabajo-Ayala; María B. Alarcón-Valencia

adquiere consistencia uniforme, se vierte en una máquina de moldear ladrillos o bloques, según elemento constructivo deseado. (Gaggino, Arguello , \& Berretta , 2008)

Para la recolección de los datos se elabora el diseño de la mezcla para bloque de 7x20x40 cm y de 10 x 20x40 con dos celdas de aire, es decir de un acabado rustico debido a las partículas (granulometría) de la materia prima, es de color gris grisáceo natural.

\section{Criterios éticos}

En consecuencia con los conocimientos adquiridos en mi profesión de Ingeniero Civil y como maestrante se aplica los criterios para la elaboración de estudio de caso.

El estudio de caso se lo desarrolla de manera estructurada, seleccionando la materia prima para la fabricación de un bloque con el chip de plástico y así poder establecer el costo y poder compararlo con el bloque tradicional, y cumplir con las Normas Técnicas Ecuatorianas INEN.

\section{Resultados.}

Una vez realizado el diseño de los componentes y las proporciones para la elaboración del bloque, el proceso, el curado se obtiene un bloque de mampostería para vivienda que sea resistente a la intemperie, baja absorción de agua, aislante térmico, liviano, buena resistencia mecánica y principalmente ecológico. El resultado obtenido dependerá mucho de cumplir con el diseño de mezcla, cumplir con el proceso de fabricación, cumplir con las especificaciones técnicas NTE INEN 638 y NTE INEN 643. 


\section{Discusión.}

De acuerdo a los datos y la bibliografía revisada y obtenida con respecto a la contaminación por desechos sólidos, los plásticos representan aun un problema medio ambiental que debe ser atendido, al ser un producto industrializado, se puede pensar en convertir un aspecto negativo como es la duración del plástico al deterioro por muchos años, en aprovechar las propiedades del PET para reutilizar como materia prima para la fabricación de bloques de pared para construir viviendas y además de contribuir con un solución ecológica aportar con una alternativa al déficit de vivienda.

El proceso de la obtención del chip a partir de las botellas de PET, esta difundido en algunos países, incluso en nuestro país en la provincia del Guayas, ya funcionan plantas procesadoras del PET para obtener el CHIP de Plástico.

En continuidad el chip de plástico es la única materia prima diferente para la fabricación del bloque ya que los demás son los componentes tradicionales como arena, cemento y aditivos que se mezclan en las proporciones de acuerdo al diseño obtenido.

Una vez obtenido en producto final a través del respectivo análisis se puede concluir si se obtiene un ahorro en el costo del bloque o se trata de una solución ecológica que reutilizará un desecho sólido que afecta al medio ambiente por la dificultad para ser absorbido por la naturaleza.

\section{Conclusiones.}

Una vez realizado todo el proceso de la fabricación del bloque pasando por la obtención del chip de plástico a partir de las botellas de plástico y que al usarse aproximadamente 1000 bloques por vivienda unifamiliar de aproximadamente $48 \mathrm{~m} 2$, diseñada por el MIDUVI, para el programa del Bono de la vivienda, consume $600 \mathrm{~kg}$ de chip de plástico, que para obtener tal 
Judy M. Flores-Cherrez; Brick L. Reyes-Pincay; Saturnino A. Carabajo-Ayala; María B. Alarcón-Valencia

cantidad de materia prima se requieren triturar 9000 botellas, se concluye que la utilización del PET como material alternativo de la construcción es ecológicamente viable como medida medioambiental. Con respecto a lo económico se concluye también que el proceso debe ser industrializado para obtener materia prima barata, para la fabricación del BLOQUE CON CHIP DE

\section{PLASTICO.}

\section{Bibliografía.}

Aguilar, Malena (2016). Agotamiento de los recursos naturales. Chile: El Centro de Tesis, Documentos, Publicaciones y Recursos Educativos

Alcívar Cordova, EV. (2015). Manejo de los desechos solidos en la provincia de el oro y su impacto ambiental en los ecosistemas. Machala: Universidad Tecnica de Machala.

Barrera, L. A. (20 de Agosto de 2015). El mundo de los plásticos. Obtenido de http://elmundodelosplasticoxd.blogspot.com/

Flake Wash. (2015). ¿Qué se puede hacer con el PET reciclado?: Mexico: Alta Tecnología Quimíca

Gaggino, R. (Octubre de 2008). Obtenido de Componentes constructivos elaborados con una mezcla cementicia $y$ agregados de rlásticos reciclados: http://uniciencia.ambientalex.info/infoCT/Comconelamezcemagrplarecar.pdf

Gaggino, R., Arguello , R., \& Berretta , H. (2008). Aplicación de material plástico reciclado en ele-mentos constructivos a base de cemento. IV Encontro Nacional e II Encontro Latino-americano sobre Edicaciones en edificacion sustentables , 906-915

INEC. (2010). Resultados del Censo 2010 de población y vivienda en el Ecuador. Quito: Instituto Nacional de Estadística y Censos

Mendieta, E. R. (2016). Diseño de mezcla de Hormigón simple de $240 \mathrm{~kg} / \mathrm{cm} 2$ utilizando Fibras de Materiales Reciclados (Botellas de Plástico). Cuenca: UCACUE.

Ministerio del Ambiente. (2012). Programa "PNGIDS" Ecuador. Obtenido de Programa Nacional para la Gestión Integral de Desechos Sólidos - PNGIDS ECUADOR:

Zavala, G. (2015). Diseño y desarrollo experimental de materiales deconstrucción utilizando plástico reciclado. Escuelaespecializadaeningenieríaitca-FEPADE, 1-45. 